\title{
Patrimonialización y gestión del territorio en la triple frontera de Brasil, Argentina y Paraguay: continuidades y desafíos del parque Iguazú ${ }^{1}$
}

\author{
Gisela Aquino Pires do Rio² y Leo Name ${ }^{3}$
}

\begin{abstract}
RESUMEN
Este artículo trata del proceso de patrimonialización de espacios y recursos naturales en el área transfronteriza que se encuentra entre Argentina, Brasil y Paraguay. Insistimos en el hecho de que la condición de Triple Frontera determina superfícies de regulación en función de acuerdos e intereses vinculados a los países limítrofes. En esta triple frontera tres formas distintas de regulación sectorial son las que establecen el patrón de uso de la tierra: la urbana, la ecológica y la eléctrica. Como primera aproximación presentamos a la patriminialización como un dispositivo de regulación ambiental. Para ello se realizó un relevamiento de información sobre las áreas de protección ambientales que se localizan en los limites de estos tres países. Dicha información revela claramente dos posibilidades en términos de uso y preservación de los recursos naturales, ambas relacionadas a la gestión de las aguas. Los ejemplos de las unidades brasileña y argentina del Parque Iguazú indican una estrategia orientada hacia la seguridad hídrica.
\end{abstract}

Palabras clave: espacios protegidos transfronterizos, Triple Frontera, gestión del territorio, integración sudamericana.

\begin{abstract}
This paper examines the patrimonialisation process of spaces and natural resources at transboundary area between Argentina, Brazil and Paraguay. We insist on the fact that the condition of Triple Border determines regulation surfaces based on arrangements and interests linked to the neighbouring countries. On this Triple Border, three differents ways of regulation set patterns of land use: urban, ecological and electric. As a first approximation, we present patrimonialization as a environmental regulation instrument. Therefore, collecting and processing data were done about environment protected areas that are located at the edges of these these three countries. The data clearly reveals two options of natural resources use and conservation, both related to water management. The examples of Brazilian and Argentinean conservation units of Iguazú Park indicate a strategy oriented by hydric safety.
\end{abstract}

Keywords: transboundary protected areas, Triple Frontier, territorial management, South America integration.

Artículo recibido el 6 de junio de 2016, aceptado el 23 de septiembre de 2016 y corregido el 20 de diciembre de 2016 Instituto de Geociencias, Universidad Federal de Rio de Janeiro (Brasil). E-mail: gpiresdorio@gmail.com

Instituto Latinoamericano de Tecnología, Territorio e Infraestructura, Universidad Federal de Integración Latinoamericana (Brasil). E-mail: leonardo.name@unila.edu.br 
Uno de los mecanismos de cooperación internacional que ha despertado un gran interés entre autores de diferentes disciplinas ha sido la implantación de las áreas protegidas en zonas de fronteras internacionales o cercanas a ellas. Tales mecanismos ponen de manifiesto cómo los procesos de regulación y gestión, la adopción de unidades territoriales de planeamiento y la formación de superficies de regulación, en cada lado de la frontera, pueden permanecer sincrónicos y estáticos y cómo los países pueden permanecer reticentes a la implantación de institucionalidades específicas que involucren decisiones y soberanías compartidas.

Nos centramos en una de las triples fronteras sudamericanas: la frontera entre Brasil, Argentina y Paraguay, en la región que se conoce como Alto Paraná, donde existe un área urbana continua formada por las ciudades de Foz do Iguaçu (en el estado de Paraná, Brasil), Puerto Iguazú (en la provincia de Misiones, Argentina) y Ciudad del Este (capital del departamento Alto Paraná, Paraguay). Allí, la naturaleza, objeto de protección, se institucionalizó bajo la forma de unidad de conservación en cada país y como patrimonio mundial de la UNESCO: las porciones argentina y brasileña del Parque Iguazú. Se trata de un área que posee agua en abundancia, destino del turismo regional e internacional, y que, desde que se construyó la usina hidroeléctrica Itaipu Binacional, es conocida también por su rendimiento hidroenergético.

Pretendemos contribuir al debate esbozando dos cuestiones. La primera: creemos que en muchos trabajos relacionados con la misma problemática el énfasis recae sobre la noción de seguridad ambiental, y en este sentido es muy poco lo que se investiga sobre las situaciones de abundancia de recursos hídricos. La segunda: creemos que la discusión sobre Áreas Protegidas Transfronterizas (APT) debe contemplar necesariamente un análisis transescalar de los fenómenos naturales, considerando, por lo tanto, el factor urbano que incide sobre ellas, sea por la presencia de las ciudades de frontera (o ciudades gemelas); por los múltiples usos y apropiaciones de la naturaleza en las ciudades; por las diferentes y variadas movilidades en las ciudades y entre las ciudades; o finalmente por las mallas de regulación formadas en cada país con miras al planeamiento y a la gestión de los espacios urbanos y las unidades de conservación.

Sin ánimos de brindar respuestas definitivas, las preguntas que guían nuestro interés en la investigación son las siguientes: ¿qué convergencias y divergencias entre normas, formas y superficies de regulación se pueden identificar? ¿Son la simetría y la sincronía de acciones en las áreas transfronterizas condiciones necesarias para las nuevas institucionalidades? ¿Influencian en la gestión urbana y ambiental de los espacios transfronterizos? Estas preguntas nos orientan hacia una investigación más amplia sobre la dinámica transfronteriza. Insistimos en el hecho de que la condición de Triple Frontera determina superficies de regulación en función de acuerdos e intereses vinculados a los países limítrofes. En esta triple frontera, tres distintas regulaciones sectoriales son las que establecen el patrón de uso de la tierra: la urbana, la ecológica y la eléctrica. En esta primera aproximación nos confrontamos con la regulación ambiental, empleando para ello el ejemplo del Parque Nacional de Iguaçu y el del Parque Iguazú.

\section{Premisas}

Nuestra principal premisa consiste en considerar al territorio como institución, o sea, tanto producto como sujeto a la regulación social, económica, política y ambiental, cuya dinámica geoinstitucional - interacción entre individuos, organizaciones y Estado - se encuentra atravesa- 
da por la tensión entre la regulación social, económica y técnica para el control y acceso a los recursos, por un lado; y por la regulación política para el dominio y control, por el otro. Argumentamos que el proceso de patrimonialización del espacio, en ese sentido, tiende a modificar el nivel de gobernanza en las fronteras, a permitir que surjan nuevas institucionalidades y a presionar para que se realicen negociaciones más participativas.

Nuestra segunda premisa resalta que comprender la frontera en la contemporaneidad requiere comprender la formación de los espacios transfronterizos. Existen diversos e importantes estudios sobre el tema de las fronteras desde la década de 1950. Su evolución nos muestra la variedad de interpretaciones y significados que ha asumido el término: vinculado al control y asociado a la idea de seguridad del territorio nacional y por lo tanto al dominio del Estado; a la ampliación del espacio económico de determinado país y al potencial de explotación de los recursos (Becker, 1982); a las fronteras-corredor (Ciccolella, 1997); a la confrontación frente a la expansión capitalista (Martins, 2014); a los nuevos patrones de funcionalidad del limite y de la frontera y a la formación de territorios-objeto para cumplir objetivos específicos de seguridad o de naturaleza económica (Machado, 2000); a las regiones ricas en recursos naturales cuya valoración por parte del mercado internacional es tributaria de la economía extractiva (Bunker, 1985); y, finalmente, a la localización, extensión y dinámica de cadenas de commodities minerales (Coelho \& Wanderley, 2013). Cada uno de esos aspectos incluye, aunque no sea de modo explícito, interacciones y flujos marcados por circuitos legales e ilegales y por una mayor o menor porosidad de las fronteras (Machado, 2002).

Las connotaciones que el término frontera adquiere en los estudios geográficos son amplias. Reboratti (1990) definió frontera como "área de transición entre el territorio utilizado y poblado por una sociedad y otro que, en un momento particular del desarrollo de esa sociedad y desde su punto de vista, no ha sido ocupado en forma estable, aunque sí puede haber sido utilizado esporádicamente", reforzando la importancia de la frontera en la valorización de los recursos naturales, en la organización del territorio y en el desarrollo de los países del continente americano.

Nuestra última premisa es la afirmación de que para comprender la formación de los espacios transfronterizos es necesario un abordaje que amplíe y dinamice los significados respecto a la escala. Diferentes escritos señalan, por un lado, que las diferencias de tamaño y de posicionamiento frente a un objeto modifican necesariamente su análisis e interpretación (Castro, 1995; Corrêa, 2002-04). Por otro lado, indican que la escala no hace referencia solamente al tamaño escogido para representar un objeto, o a la extensión espacial de los fenómenos (global, nacional, regional, local), sino también a los niveles de análisis a través de los cuales ocurre un proceso relevante. Finalmente, como constructo social, la escala articula tanto sincronía como diacronía en la comprensión de fenómenos (Egler, 1990; Goodchild \& Quattrochi, 1997; Marston, 2000; Smith, [1992] 2002).

A partir de estas premisas comprendemos que el espacio geográfico posee una mayor complejidad que la capacidad de explicación de distintas y aisladas escalas y que sobretodo en espacios transfronterizos el análisis difícilmente pueda restringirse a las unidades territoriales impuestas por el Estado u otras instituciones. Se trata de espacios donde emanan conflictos de la interacción y de la movilidad de los fenómenos, personas y objetos frente a límites territoriales rígidos: continuidades y discontinuidades espaciales componen sus superficies de regulación. Son un enorme desafío que involucra también áreas protegidas y recursos compartidos. 


\section{Áreas protegidas transfronterizas y patrimonio natural}

Las acciones que intervienen en la creación de unidades de conservación y de espacios protegidos pueden ser resultado de la presión ejercida por diferentes agentes y organizaciones. La Unión Internacional para la Conservación de la Naturaleza (UICN) y diferentes convenciones Internacionales (Patrimonio Mundial, RAMSAR, Reserva de Biosfera) ilustran las modalidades de presión ejercidas para la constitución de espacios protegidos y unidades de conservación de varias categorías y con usos más o menos restringidos.

Para la UICN las áreas protegidas transfronterizas son espacios claramente definidos, reconocidos y gestionados a partir de medios eficaces (jurídicos u otros), con miras a asegurar la conservación de la naturaleza a largo plazo (Dudley, 2008). Para descomponer esta definición es necesario considerar la relación diacrónica entre espacio y tiempo. "El espacio claramente definido" remite al "nomos" como acción que delimita, crea superficies reguladas de diferente forma al espacio que las rodea (Pires do Rio, 2011). "Asegurar la conservación a largo plazo" insiste sobre la dimensión temporal: garantizar las condiciones de transmisión del objeto que se quiere legar a las generaciones futuras; de esta forma, el énfasis está en la transmisión de aquello que fue elegido como legado y en reforzar su protección a escala local y global. Se acentúa por lo tanto la necesidad de reconocer los límites que las demarcan y la diacronía de la función que se les atribuye.

Al mismo tiempo, hay dos lógicas enfrentadas: la lógica de la gestión compartida de fracciones del espacio, y por lo tanto, de la estructura específica para dicha finalidad; y la lógica de las organizaciones supranacionales de diversos tipos que se encargan de esta tarea - y que pueden afectar, influenciar, determinar o condicionar la apropiación y explotación de los recursos involucrados. En ambos casos estamos frente a la constitución de stocks y reservas - tierras, agua y demás elementos que pueden llegar a ser patrimonializados como paisajes, lugares y especies (Castro, 2013) - cuyo régimen de gestión impone importantes desafíos.

En ese sentido nos interesa cuestionar qué es patrimonio y por qué. En el debate académico Brunet et al. (1992: 335) ampliaron la concepción de patrimonio natural o genético: para ellos la herencia que trae cada ser vivo constituye una fuente de patrimonio, enfatizando desde este punto de vista la creación de valores fundamentales como elemento central en la patrimonialización de determinados objetos, áreas o aspectos de la vida. Lacoste (2003), por su parte consideró que es posible ampliar el sentido original del término si se designa al conjunto de bienes que pertenecen a un sujeto colectivo, asumiendo entonces una función identitaria. Y finalmente, para Castro y Zusman (2007), el proceso de patrimonalización implica la construcción de una red escalar que aproxime actores situados en distintos lugares: esto significa el acercamiento de los actores que operan en diferentes escalas y que pueden efectivamente mediar entre las relaciones locales y globales como proceso dialéctico.

La noción de patrimonio natural con implicaciones institucionales fue difundida a partir de la XVII Conferencia de las Naciones Unidas para la Educación y la Cultura, realizada en 1972. Veinte años más tarde la Convención de Biodiversidad contribuyó a la ampliación de la noción de patrimonio natural cuando reconoció el valor intrínseco de la diversidad biológica, reconociendo también que su conservación es una preocupación común de la humanidad y que es necesario determinar quiénes serán los signatarios encargados de conservar y utilizar la diversidad biológica en favor de las futuras generaciones. Contribuyó también a instituir la demarcación de áreas 
asociadas a la protección de la naturaleza a través de la creación de zonas delimitadas geográficamente que son designadas, reglamentadas y administradas teniendo en cuenta los objetivos específicos de conservación. Se puede decir entonces que las categorías de áreas protegidas y los criterios para describir los tipos de patrimonio evolucionan de modo paralelo (Cuadro No 1): como sistemas de protección que inciden sobre las áreas de forma sucesiva y concomitante, suscitando un importante número de preguntas. Se puede incluir a los parques nacionales en los criterios I, II y IV, ya que su definición incluye un área extensa de gran relevancia para la biodiversidad y para la protección ecológica. Es importante resaltar que, inspirados en la UICN, Brasil y Argentina consideran para la delimitación de Parques Nacionales la relevancia ecológica y la belleza paisajística bajo el control del Estado.

Cuadro No 1

Criterios de definición del Patrimonio Natural y categorías para las Áreas de Protección

\begin{tabular}{|c|c|c|c|}
\hline \multicolumn{2}{|r|}{ Patrimonio Natural } & \multicolumn{2}{|c|}{ Áreas de Protección } \\
\hline Criterios & Descripción & Categorías & Descripción \\
\hline I & $\begin{array}{l}\text { Representar fenómenos naturales o áreas de } \\
\text { belleza natural e importancia estética excep- } \\
\text { cionales. }\end{array}$ & I & $\begin{array}{l}\text { Reserva natural/Silves- } \\
\text { tre }\end{array}$ \\
\hline II & $\begin{array}{l}\text { Grandes etapas evolutivas de la historia de la } \\
\text { tierra que comprenden: testimonios de vida, } \\
\text { procesos geológicos en el desarrollo de for- } \\
\text { mas terrestres o elementos geomorfológicos } \\
\text { o fisiográficos de gran significado. }\end{array}$ & II & Parque Nacional \\
\hline III & $\begin{array}{l}\text { Procesos ecológicos y biológicos en la evo- } \\
\text { lución de los ecosistemas y comunidades de } \\
\text { plantas y de animales terrestres, acuáticos, } \\
\text { costeros y marinos. }\end{array}$ & III & Monumento Natural \\
\hline \multirow{3}{*}{ IV } & \multirow{3}{*}{$\begin{array}{l}\text { Contener los "hábitats" naturales más repre- } \\
\text { sentativos y más importantes para la con- } \\
\text { servación in situ de la diversidad biológica, } \\
\text { incluyendo a aquellos "hábitats" donde so- } \\
\text { breviven las especies amenazadas, con valor } \\
\text { universal excepcional del punto de vista de la } \\
\text { ciencia o de la conservación. }\end{array}$} & IV & $\begin{array}{l}\text { Áreas de manejo de há- } \\
\text { bitats o especies }\end{array}$ \\
\hline & & V & $\begin{array}{l}\text { Paisaje Terrestre o Mari- } \\
\text { no Protegido }\end{array}$ \\
\hline & & $\mathrm{VI}$ & $\begin{array}{l}\text { Área Protegida con Re- } \\
\text { cursos Manejados }\end{array}$ \\
\hline
\end{tabular}

Fuente: UNESCO (1972) y UICN (1992).

Antes de la Conferencia de 1972 la noción de patrimonio de la humanidad ya había sido empleada en los textos preparatorios de la Convención de las Naciones Unidos sobre el Derecho del Mar, que tuvo lugar recién en 1982. Hasta esa fecha se habían realizado otras dos reuniones para tratar el tema del patrimonio: Ginebra en 1958 y Malta en 1967. Lo que nos interesa particularmente es el hecho de que desde comienzos de la década de 1970 se empleaba el término "patrimonio común de la humanidad" sin estar asociado en aquel momento a una malla institucional específica - sea administrativa o política. Hubo un cambio importante en relación a ese periodo que hace 
con que Wolmer (2003), por ejemplo, considere un gran desafío la gestión de las áreas protegidas delimitadas a través de fronteras administrativas e internacionales.

La constitución de Áreas Protegidas Transfronterizas remite al contexto de las décadas de 1920 y 1930, donde funcionó como un mecanismo de resolución de disputas por la fijación de límites entre países. Las dos primeras áreas que fueron creadas estaban sujetas a este tipo de problema: el Parque de la Paz Glaciar-Waterton, entre Canadá y Estados Unidos, y en la frontera entre Polonia y la antigua Checoslovaquia la asociación del Polish Pieniny National Park y la Slovak National Natural Reserve dieron origen a un segundo Parque de la Paz (Protocolo de Cracovia, de 1924, implementado en 1932) (Steiman, 2008).

En 1933 la Convención para la conservación de la flora y la fauna de Londres estableció el principio de protección transfronteriza y de cooperación en las zonas donde se propusiera crear parques o donde ya estuvieran implantados. En gran medida esa Convención trató de incorporar la noción de ecosistema como aquella que se impone a la creación de este tipo de unidad de conservación. De hecho, formas como las de corredores y mosaicos abarcan áreas contiguas que pertenecen a distintos regímenes de regulación en la esfera nacional. Son parques, reservas o bosques que en su calidad de unidades de conservación transfronterizas y teniendo la función de favorecer la circulación de la fauna silvestre y asegurar la integridad de los ecosistemas llevan a cabo políticas y prácticas de conservación que requieren necesariamente sincronicidad en las regulaciones por parte de los países limítrofes. La adopción de unidades ecológicas de base y la delimitación de Áreas Protegidas Transfronterizas se encuentran en tensión porque implican nuevas lógicas de actuación y de tomada de decisiones, así como nuevas tensiones en los campos científico y geopolítico, en la adopción de sistemas de gobernanza y de tomada de decisiones de arriba hacia abajo (top-down) y de abajo hacia arriba (bottom up) (Wolmer, 2003) y en la gestión de recursos compartida. Las Áreas Protegidas Transfronterizas engloban subcategorías como parque de la paz, además de reflejar designaciones relativas a otras convenciones que inciden sobre las unidades nacionales, como sitios RAMSAR y Reservas de Biosfera y Patrimonio Natural, por ejemplo. Estas designaciones permiten ampliar el espacio sujeto a los criterios de preservación y conservación.

Es posible por lo tanto establecer un paralelismo entre las Áreas Protegidas Transfronterizas y la noción de patrimonio, en la medida en que todo aquello que se constituye como patrimonio es resultado de una construcción social, sin que existan entonces características intrínsecas a cualquier objeto, elemento o ser vivo que lo califique como patrimonio "en si" (Ost, 2003). Como noción de fuerte carga operacional en el dominio de la gestión del territorio, las Áreas Protegidas Transfronterizas reflejan la constitución de stocks para necesidades proyectadas y les atribuye una finalidad actual y futura a recursos potenciales y efectivos. Regular y controlar al mismo tiempo el uso actual y asegurar la transmisión, optar entre lo que será transmitido respetando la diversidad seleccionada: esos parecen ser los elementos que constituyen la paradoja de la naturaleza-patrimonio y de la gestión compartida en las regiones fronterizas.

\section{Áreas protegidas en zonas fronterizas de América del Sur}

La patrimonialización de las Áreas Protegidas Transfronterizas, a nuestro modo de ver, implica una doble protección: el patrimonio colectivo y una situación particular que requiere sincronicidad en las decisiones regulatorias entre países limítrofes. 
Para reforzar este argumento retomamos algunas definiciones sobre las particularidades de los espacios transfronterizos en el contexto sudamericano (Laurelli, 2004; Laurelli e Schweiter, 2005; Pires do Rio, 2011). La dinámica de estos espacios se encuentra marcada por los cambios institucionales que en el proceso de integración regional se proyectaron a mayor escala dada la intensificación de las interacciones espaciales, los flujos de personas, mercaderías, capital, servicios, energía e información, de naturaleza legal e ilegal; y por el aumento de acciones de cooperación interregionales que son al menos binacionales. Son espacios que aún no encontraron un cuadro institucional que los regule como tal y cuya dinámica es aprendida a escala regional, bajo el efecto de cuadros reguladores distintos (Pires do Rio, 2011) e institucionalidades igualmente distintas. Destacamos el hecho de que la condición transfronteriza es constitutiva de la dinámica regional, sea por la exigencia de normas y reglas particulares como aquellas que inciden sobre las unidades de conservación, los recursos hídricos y el rendimiento hidroeléctrico, o por el enfrentamiento de estas reglas en el caso de los circuitos ilegales o incluso por los movimientos cotidianos y pendulares.

Una característica que parece predominar en las Áreas Protegidas Transfronterizas en América del Sur es la divergencia que existe entre la contigüidad del ecosistema y la gestión compartida o la división de la soberanía sobre parte del territorio. En este sentido, hay diferencias entre la creación de espacios protegidos demarcados de manera sincrónica, de un lado u otro del límite internacional y aquellos de gestión compartida. En varias configuraciones fronterizas, de norte a sur, se puede observar la implantación de unidades de conservación definiendo un patrón simétrico de uso de la tierra sin correspondencia en términos de gestión compartida de estas unidades. Si por un lado la dimensión ecosistémica y ecológica permite acentuar la unidad de los diversos mosaicos, el control y la gestión permanecen como dimensiones de la esfera estatal.

Cabe destacar dos ejemplos en áreas de frontera poco pobladas. El primero constituye uno de los casos más significativos de América del Sur: se trata del Parque Cóndor en el área transfronteriza entre Ecuador y Perú. En 1998 como resultado del tratado de delimitación de frontera firmado en Brasilia se establece una zona de protección ecológica que será instituida a través de la creación de parques nacionales contiguos (2.540 hectáreas en Ecuador y 5.440 hectáreas en Perú). Además de los respectivos parques nacionales - Parque Nacional Ichikag, Muja Cordillera del Cóndor, en Perú; y Refugio de la Vida Silvestre El Zarza, Reserva Biológica El Quimi y el Parque Binacional El Cóndor, en Ecuador - se creó el Parque de la Paz en la frontera conocida como Alto Cenepa. La institución de esta unidad de conservación permitió la reaproximación de grupos étnicos como los Achuar en Ecuador y los Aguarunas en Perú. En lo que concierne a la gestión de estas unidades aún permanece el vínculo institucional exclusivo con los respectivos Estados.

El segundo ejemplo es el de la triple frontera entre Chile, Argentina y Bolivia, donde los respectivos parques nacionales no llevaron adelante iniciativas de gestión compartida a pesar de los importantes intercambios en el sector turístico. No constituyen estrictamente un Área Protegida Transfronteriza: por el contrario, los mecanismos de protección son utilizados para la afirmación nacional - la ampliación y la superposición de las convenciones tienen por objetivo reforzar las singularidades del entorno de cada una de las unidades. La Quebrada de Humahuaca en la provincia de Jujuy (Argentina) fue elevada a la categoría de Paisaje Cultural de la Humanidad en 2002; en San Pedro de Atacama, en Chile, se creó en 1990 la Reserva Nacional Los Flamencos; y finalmente en Bolivia se demarcó (en 1973) y amplió (en 1981) la Reserva Nacional de Flora y Fauna Eduardo Avaroa. Dentro de este mosaico el turismo funciona como el principal eje de interacción 
entre las reservas, reafirmando, como ya fue mencionado, el principio de soberanía de cada Estado en la gestión de las unidades de conservación.

Cuadro No 2

Brasil: Unidades de Conservación Federales en regiones de frontera

\begin{tabular}{|l|c|c|r|}
\hline Unidad de Conservación & País limítrofe & $\begin{array}{c}\text { Año de } \\
\text { Creación }\end{array}$ & \multicolumn{1}{c|}{ Área (ha) } \\
\hline Parque Nacional do Iguaçu & Argentina & 1939 & $169.695,88$ \\
\hline Reserva Biológica Guaporé & Bolivia & 1982 & $615.771,56$ \\
\hline Parque Nacional do Pantanal Matogrossense & Bolivia & 1981 & 135.000 \\
\hline Reserva Extrativista Chico Mendes & Perú & 1990 & $931.537,14$ \\
\hline Floresta Nacional de Santa Rosa do Purus & Perú & 2001 & $231.555,52$ \\
\hline Reserva Extrativista Alto Juruá & Perú & 1990 & $537.946,47$ \\
\hline Estação Ecológica Rio Acre & Perú & 1981 & $77.500,00$ \\
\hline Parque Nacional da Serra do Divisor & Perú & 1989 & $837.555,19$ \\
\hline Parque Nacional Pico da Neblina & Venezuela & 1979 & $2.252 .616,84$ \\
\hline Floresta Nacional Amazonas & Venezuela & 1989 & $1.573 .100,00$ \\
\hline Parque Nacional do Monte Roraima & Venezuela/Guyana & 1989 & $116.747,80$ \\
\hline Parque Nacional do Tumucumaque & Surinam/Guayana & 2002 & $3.865 .188,53$ \\
\hline Parque Nacional do Cabo Orange (mar) & Guayana Francesa & 1980 & $657.318,06$ \\
\hline Total & & & $12.001 .532,99$ \\
\hline
\end{tabular}

Fuente: ICMBio. No se incluyeron las unidades que a pesar de estar localizadas en zonas de frontera no limitan con otros países.

En Brasil, dadas sus dimensiones continentales, la Constitución Federal (Brasil, 1988) estableció oficialmente una franja de $150 \mathrm{~km}$ de radio a partir de sus límites hacia el oeste con los demás países y hacia el este con su interior: todos los municipios interceptados en esta franja forman parte de su "zona de frontera" (Brasil, 2009). Dentro de esta franja se localizan trece de las unidades de conservación que comparten sus límites coincidentes con los países vecinos (Cuadro No 2).

Una de estas unidades de conservación -el Parque Nacional do Iguaçu- es contigua a otra unidad en el lado argentino. Las dos unidades poseen 252.880 hectáreas, casi 170.000 hectáreas delimitadas del lado brasileño. En Argentina se creó el parque en 1934, a partir de la Ley $\mathrm{N}^{\circ}$ 12.103 (Argentina, 1934) y fue elevado a la categoría de Patrimonio Natural de la Humanidad por la UNESCO en 1984. El parque brasileño se creó en 1939 a través del Decreto No 1.035 (Brasil, 1939), sus límites fueron definidos cinco años más tarde mediante el Decreto 6.664 (Brasil, 1944) y se convirtió en patrimonio natural de la UNESCO en 1986. Es importante notar, por lo tanto, que pese a que se destinó un tiempo a los ajustes legales - primero para legitimar los límites, después para refrendar la decisión de la Convención - hubo sincronicidad en las acciones de los dos países. 
No podemos pasar por alto el hecho de que esta región representa para los argentinos las áreas húmedas que aún hoy no recibieron la denominación de litoral. Un corredor fluvial de innegable importancia geo-histórica, pero cuyo análisis escapa a los objetivos de este trabajo. Lo que destacamos, de todas formas, es la asimetría en la demarcación de áreas protegidas en esta triple frontera. Esta particularidad se explica porque este corredor constituye la principal fuente de agua dulce de Argentina. Entre las iniciativas que existen a escala local, como veremos más adelante, encontraremos similitudes.

\section{El parque Iguazú: continuidades y desafíos}

El Parque Iguazú constituye un caso singular para el análisis de las Áreas Protegidas Transfronterizas, ya que se encuentra en un área de ocupación reciente - Puerto Iguazú en Argentina, Foz do Iguaçu en Brasil, y Ciudad del Este en Paraguay, fundadas oficialmente en 1902, 1914 y 1957 respectivamente - densamente poblada y en un contexto de gran abundancia de recursos hídricos.

Completan esta singularidad la formación de parques en un período relativamente temprano en relación a las preocupaciones ambientales en los países de América del Sur y también su considerable participación en las actividades económicas y redes de turismo de la región. Foz do Iguaçu, por ejemplo, tiene la tercera posición en el ranking de los destinos más visitados por extranjeros en Brasil y las motivaciones que llevan a los turistas a visitarla están vinculadas a los atractivos de las porciones brasileña y argentina del parque Iguazú. Ambas tuvieron más de 1,5 millón de visitantes (extranjeros y domésticos) en 2012 y un incremento de cerca del $10 \%$ en relación al año anterior, números que hacen del parque nacional argentino el más visitado del país y del brasileño el segundo más visitado (Argentina, 2013; Brasil, 2013).

Además de eso, el parque se encuentra sujeto directa o indirectamente a los regímenes de regulación territorial de los países, propiciando el debate sobre las configuraciones físicas de los territorios, sus dinámicas urbanas, los diferentes órdenes jurídicos, las diversas superficies y mallas de regulación de los recursos naturales (particularmente del agua y de los bosques) y las consecuentes divisiones administrativas con diferentes unidades espaciales de planeamiento con variados niveles de interacción y cooperación.

Destacamos dos continuidades correlacionadas en la Figura No 1. Primeramente, la urbana: separadas por los cursos de los ríos Paraná e Iguaçu pero conectadas a través de puentes y un fácil acceso- gracias a los acuerdos sobre movilidad entre los tres países -las tres ciudades forman una conurbación con una dinámica propia en relación al flujo de personas, redes y división de funciones derivadas del turismo y de la instalación de equipamientos como la usina binacional de Itaipu.

Existe también una continuidad a la cual vamos a dar más destaque: la medioambiental. En la medida en que las tres ciudades, los dos parques y los tres países son atravesados por los rios Iguaçu y Paraná, y en estas ciudades es común el registro de inundaciones, sobretodo en áreas de ocupación irregular por poblaciones de bajos recursos en los márgenes o cercanías de estos ríos, se vuelve importante indagar sobre las relaciones complementarias o contrastantes entre el régimen hídrico - la dinámica de crecidas y estiajes de los ríos y su asociación con el clima 
Figura No 1

Porciones brasileña y argentina del Parque Iguazú y conurbación urbana entre Ciudad del Este, Foz do Iguaçu y Puerto Iguazú

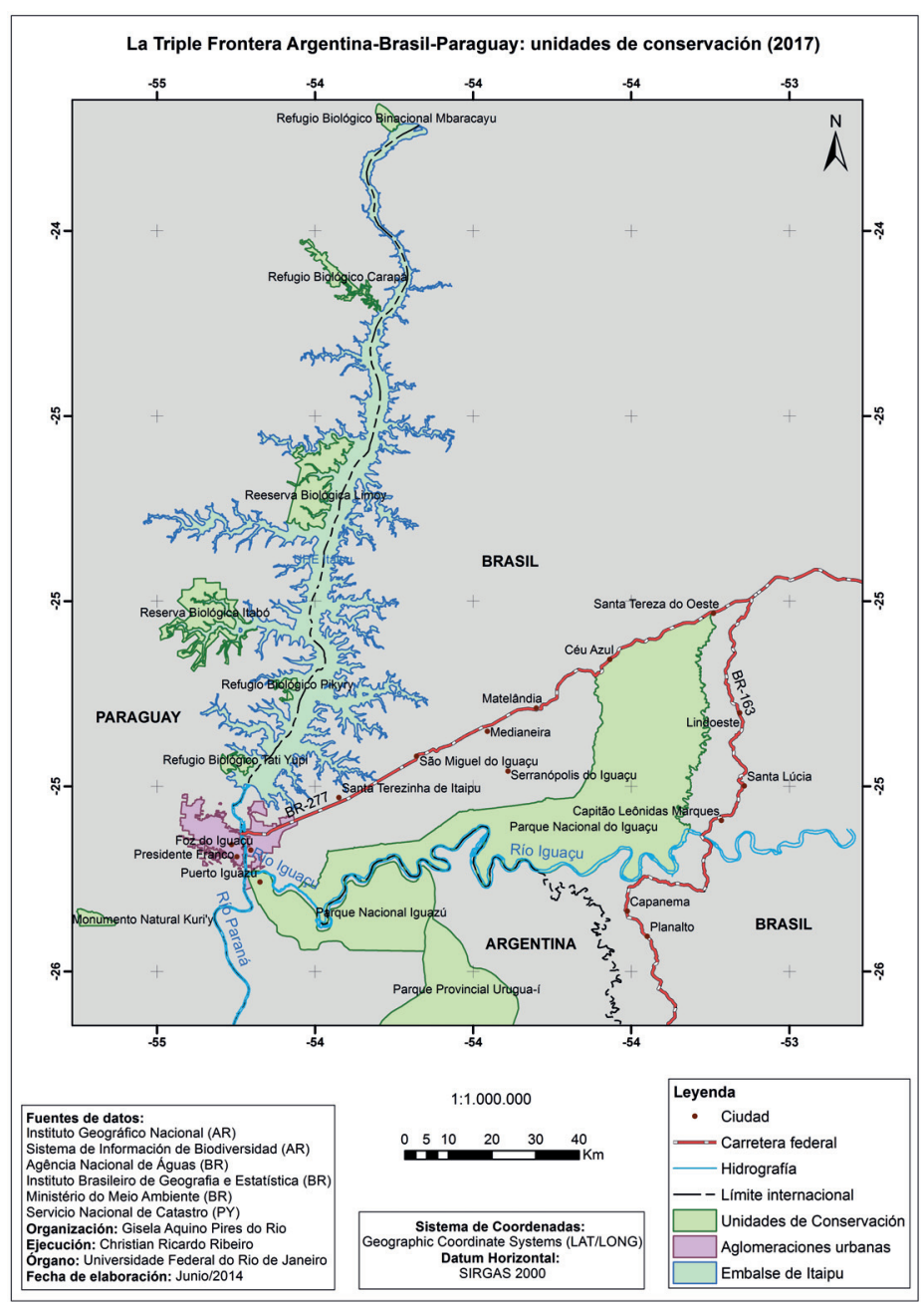

Fuente: Elaboración propia.

y períodos de lluvia más intensa, por ejemplo - y el régimen jurídico oriundo de las normativas de cada país que pretenden no solo proteger la vegetación junto a los márgenes de los ríos sino también la seguridad de las poblaciones.

El Cuadro No 3 que sigue a continuación presenta lo establecido por las leyes más recientes de cada país sobre la protección de los márgenes de los cursos de agua. Observamos distintas construcciones de áreas protegidas, de regulación nacional y con distintas geometrías.

Al comparar los Caminos de Sirga argentinos, las APP brasileñas y las Zonas de Uso Público paraguayas podemos ver que en el caso del área transfronteriza que estamos analizando los cursos de agua que establecen los límites entre un país y otro tienen cada uno de sus márgenes regulados por distintas normas de distintos países, de distintas geometrías. Se revelan así, como ya fue 
Cuadro $\mathrm{N}^{\circ} 3$

Argentina, Brasil y Paraguay: normas sobre protección de los márgenes de los ríos

\begin{tabular}{|l|l|l|l|}
\hline País & \multicolumn{1}{|c|}{ Norma } & Área protegida & \multicolumn{1}{|c|}{ Definición } \\
\hline Argentina & $\begin{array}{l}\text { Código Civil y } \\
\text { Comercial de } \\
\text { la Nación (Ley } \\
26.994 / 2014) .\end{array}$ & Camino de Sirga & $\begin{array}{l}\text { El artículo 1.974 de esta ley afirma que a lo } \\
\text { largo de los márgenes de los ríos y arroyos } \\
\text { navegables el propietario debe dejar libre } \\
\text { una zona de quince metros de ancho. }\end{array}$ \\
\hline Brasil & $\begin{array}{l}\text { Código Forestal } \\
\text { Ley 12.651/2012) }\end{array}$ & $\begin{array}{l}\text { Área de Preser- } \\
\text { vación Perma- } \\
\text { nente (APP) }\end{array}$ & $\begin{array}{l}\text { Del conjunto de los espacios caracteriza- } \\
\text { dos en el Código Forestal como APP, en su } \\
\text { artículo 40, inciso I, establece que consti- } \\
\text { tuyen áreas de preservación permanente } \\
\text { las franjas marginales de cualquier curso } \\
\text { de agua, variando de treinta a quinientos } \\
\text { metros (dependiendo del ancho del curso } \\
\text { de agua) en las cuales el propietario debe } \\
\text { encargarse de mantener la vegetación. }\end{array}$ \\
\hline Paraguay & $\begin{array}{l}\text { Ley de Recursos } \\
\text { Hídricos del } \\
\text { Paraguay (Ley } \\
\text { 3.239/2007). }\end{array}$ & Zona de Uso Pú- \\
blico & $\begin{array}{l}\text { El artículo 23 de esta ley dice que los már- } \\
\text { genes de los cursos hídricos que sean de } \\
\text { dominio privado deben establecer una } \\
\text { franja de uso público de cinco metros en } \\
\text { zonas urbanas y de diez metros en zonas } \\
\text { rurales. }\end{array}$ \\
\hline
\end{tabular}

Fuente: Elaboración propia desde dichas normas.

señalado por Gomes et al. (2012), las contradicciones oriundas de la especificidad de los procesos ecológicos que determinan la condición de riesgo frente a los procesos de urbanización y las instancias político-jurídicas traducidas en las normas, de las cuales se derivan las áreas protegidas.

En lo que respecta a los parques, cabe mencionar que la porción brasileña del Parque Iguazú se extiende a través de catorce municipios del oeste del estado de Paraná, cada uno de ellos con diferente extensión territorial y densidad de población: Foz do Iguaçu, Santa Terezinha do Itaipu, São Miguel do Iguaçu, Medianeira, Matelândia, Céu Azul, Vera Cruz do Oeste, Santa Tereza do Oeste, Lindoeste, Santa Lúcia, Capitão Leônidas Marques, Capanema y Andresito. Del lado argentino el parque abarca apenas dos municipios: Puerto Iguazú y Puerto Libertad que forman parte del departamento de Iguazú, en la provincia de Misiones. No obstante, estas dos unidades de conservación constituyen apenas una parte diminuta de la enorme área de bosque subtropical que partiendo del parque nacional brasileño se extiende de manera fragmentaria en dirección sudoeste hasta Paraguay; y de forma continua, en dirección sur, en Misiones, ocupando cerca del $50 \%$ del área de esa provincia argentina. Además, la región Alto Paraná presenta mosaico de unidades de conservación, brasileñas y paraguayas, gestionadas por la usina Itaipu Binacional (Cuadro No 4). 
Cuadro No 4

Unidades de conservación alrededor de la Usina Itaipu Binacional

\begin{tabular}{|l|l|r|}
\hline País & \multicolumn{1}{|c|}{ Unidad de Conservación } & \multicolumn{1}{|c|}{ Área (ha) } \\
\hline \multirow{4}{*}{ Brasil } & Refugio Biológico Bela Vista & 1.920 \\
\cline { 2 - 3 } & Refugio Biológico Santa Helena & 1.482 \\
\hline \multirow{5}{*}{ Paraguay } & Refugio Biológico Carapá & 3.250 \\
\cline { 2 - 3 } & Refugio Biológico Pikyry & 1.109 \\
\cline { 2 - 3 } & Refugio Biológico Tati Yupi & 2.245 \\
\cline { 2 - 3 } & Refugio BológicoYui Rupá & 750 \\
\cline { 2 - 3 } & Reserva Biológica Itabó & 15.208 \\
\cline { 2 - 3 } & Reserva Biológica Limoy & 14.828 \\
\cline { 2 - 3 } & Reserva Natural Yvyty Rokai & 3.809 \\
\hline
\end{tabular}

Fuente: Itaipu Binacional (2016).

En lo que atañe a las áreas de protección localizadas en territorio argentino, es importante aclarar que la Constitución de la Nación Argentina (Argentina, 1994) otorga a las provincias todos los poderes que no hayan sido por ella designados como exclusivos del gobierno federal, concediéndoles bastante autonomía. Y lo más importante, la provincia tiene el control sobre los recursos naturales que se encuentran en su territorio y es en la escala provincial donde la gestión ambiental está más presente, abriendo posibilidades de articulación con provincias vecinas y quizá países vecinos.

A causa de ello, la provincia de Misiones, donde se localiza la porción argentina del Parque Iguazú, tuvo su constitución propia y revisada algunos años después de la constitución nacional (Misiones, 1998). Probablemente por estar dotada de un paisaje con una cantidad sustancial de remanentes forestales conjugados con las ruinas de las reducciones jesuíticas ha sido tratada como atractivo turístico y cuenta con una serie de leyes provinciales de diferentes periodos en lo que concierne a la regulación ambiental ${ }^{4}$. Una de las más importantes es la Ley No 3.631 que creó el Área Integral de Conservación y Desarrollo Sustentable del Corredor Verde de la Provincia de Misiones (Misiones, 1999), con 1,1 millón de hectáreas, donde se encuentra incluida la porción argentina del Parque Iguazú. Según esta ley este espacio está orientado hacia la conectividad ambiental y hacia el reconocimiento y aprovechamiento de sus servicios de ecosistema (agua limpia, fijación de carbono en la atmósfera, mantener la biodiversidad). Para ello, promueve la unión entre los principales bloques de áreas naturales protegidas de la provincia: al norte los parques provinciales de Yacuí, Urugua-í e Foerster y el Parque Iguazú; al este la reserva de biosfera Yabotí y los parques provinciales Esmeralda y Moconá; y al sur los parques provinciales Salto Encantado y Cuña Pirú. Pero las intenciones del corredor van más allá de la conexión entre estos parques ya que dentro del perímetro del corredor verde hay 31 áreas protegidas con gran cantidad de reservas privadas.

Para leer un resumen de ellas ver FUDHAM (2008) 
Esta configuración indica la imposición de corredores y mosaicos ecológicos en detrimento de cierta insularidad que dominó durante más de cincuenta años la delimitación de los espacios protegidos.

\section{Últimas consideraciones}

En un estudio más amplio que hemos realizado sobre este asunto y del cual proviene el texto que presentamos se indica que existen cuatro lógicas enfrentadas en relación a la gestión del territorio en la triple frontera: exigencias de conservación, aprovechamiento energético, demandas por infraestructura y crecimiento urbano. El parque, fuente importante de ingresos a través del turismo, es central para la economía de esta región, mientras que la usina hidroeléctrica desempeña un papel extraregional.

El interés inicial de este trabajo reside en el análisis de las relaciones entre los cambios en la regulación que impusieron una nueva dinámica a la acción de los diferentes agentes frente a los distintos dispositivos de protección medioambiental. Dentro del área de estudio los mosaicos y corredores ecológicos definen el patrón de uso de la tierra sin que los mecanismos de gestión compartida estén plenamente instituidos. En este sentido, en nuestro trabajo si por una parte se buscó resaltar que existen formas de cooperación y acciones conjuntas que han proporcionado la protección de sitios importantes para la historia sudamericana, por otra parte el patrimonio natural expresado en las porciones argentina y brasileña del Parque Iguazú refleja una necesidad de proyección de demandas regionales a escala global para la cual la articulación de las institucionalidades, con miras a una posible regulación conjunta, aún se muestra insuficiente.

Pudimos demostrar así distintos resultados en distintas escalas. Por ejemplo: la autonomía en la escala provincial, asegurada por la constitución argentina, permitió que la legislación en la escala de la provincia de Misiones haya logrado ensayar una gestión integrada de los mosaicos forestales subtropicales que existen en su territorio además del Parque Iguazú; la usina Itaipu, por su vez, por ser una institución binacional, pudo gestionar unidades de conservación en Brasil y Paraguay simultáneamente.

Sin embargo, en la legislación basada en la escala de cada uno de los países no se diseñan dispositivos que posibiliten sincronía y simetría de acciones con miras a la protección ambiental conjunta, como ejemplificaron claramente las diferencias significativas entre las áreas protegidas a lo largo de los cursos de agua - Caminos de Sirga (Argentina), Áreas de Preservación Permanente (Brasil) e Zonas de Uso Público (Paraguay).

En ese sentido, es notoria la ausencia de la figura de las Áreas Protegidas Transfronterizas como categoría específica de zonas de fronteras en el contexto sudamericano. A pesar de que la presencia de unidades de conservación a lo largo de la franja de frontera en Brasil sea significativa, el objeto de nuestro artículo ayuda a revelar que las modalidades de coordinación y gestión compartidas son poco expresivas. 


\section{Referencias bibliográficas}

ARGENTINA. Ley Nacional no 12.103. Buenos Aires, 1934.

ARGENTINA. AET 2012: Anuario estadístico de turismo. Buenos Aires: Ministério de Turismo, 2013.

ARGENTINA. Código Civil y Comercial de la Nación. Buenos Aires: Infojus, 2014.

BECKER, B Geopolítica da Amazônia. Rio de Janeiro: Zahar Editores, 1982.

BRASIL. Decreto-Ley n 1.035. Rio de Janeiro, 1939.

BRASIL. Decreto-Ley nº 6.664. Rio de Janeiro, 1944.

BRASIL. Constituição da República Federativa do Brasil. Brasília, 1988.

BRASIL. Faixa de Fronteira: Programa de Promoção do Desenvolvimento da Faixa de Fronteira. Brasília: Ministério da Integração Nacional, 2009.

BRASIL. Ley no 12.651. Brasília: 2012.

BRASIL. Anuário Estatístico de Turismo - 2013. Brasilia: Ministério do Turismo, 2013.

BRUNET, R.; FERRAS, R. \& THÉRY, H. Les mots de la géographie. Paris: La documentation Française, 1992.

BUNKER, S. Underdeveloping the Amazon. Extraction, unequal exchange, and the failure of the Modern State. Urbana: University of Illinois Press, 1985.

CASTRO, H. y ZUSMAN, P. Redes escalares en la construcción de los patrimonios de la humanidad. El caso de la patrimonialización de la Quebrada de Humahuaca (Jujuy, Argentina). GEOUSP: Espaço e Tempo, 2007, № 21, p. 173-184.

CASTRO , H. La cuestión ambiental en geografia histórica e historia ambiental: tradición, renovación y diálogos. Revista de Geografía Norte Grande, 2013, № 54, p. 109-128.

CASTRO, I.E. O problema da escala. In: CASTRO, I.E.; CORRÊA, R.L. \& GOMES, P.C.C. (Editores). Geografia: conceitos e temas. Rio de Janeiro: Bertrand Brasil, 1995, p. 117-140.

CICCOLELLA, P. Redefinición de fronteras, territorio y mercados en el marco del capitalismo de bloques. En: CASTELLO, I.R.; KOCH, M.R.; OLIVEIRA, N.; SCHÄFFER, N.O. \& STROHAECKER, T.M. (Editores). Fronteiras na América Latina: espaços de transformação. Porto Alegre: Editora da UFRGS, 1997, p. 55-67.

COELHO, M.C.N. \& WANDERLEY, L.J.M. Peru-Bolívia-Brasil: garimpeiros e ideia de região transfronteiriça. Revista da ANPEGE, 2013, Vol. 9, No 12, p. 5-16. 
CORRÊA, R.L. Uma nota sobre o urbano e a escala. Territorio, 2002-2004, N 11-12-13, p. 133-136.

DUDLEY, N. (Editor). Lignes directrices pour l'application des catégories de gestion aux aires protégées. Gland: UICN, 2008.

EGLER, C. Diacronia em três escalas. In: RIBEIRO, A.C.T. \& MACHADO, D.B.P. (Editoras). Metropolização e Rede Urbana. Brasilia. 1990, p. 147-160. Disponible en Internet: http://www.ipea.gov.br/ portal/index.php?option=com_content\&view=article\&id=7636

FUNDACION POR EL DESARROLLO HUMANO Y EL AMBIENTE (FUDHAM). Las leyes ambientales de Misiones. Posadas: Fudham, 2008.

GOMES, E.; NAME, L. \& MONTEZUMA, R.C.M. Complexidade e Conflitos: APP, espaço geográfico e espaço normativo. In: Seminário Nacional sobre Áreas de Preservação Permanente em Meio Urbano. Natal, 2012.

GOODCHILD, M.F. \& QUATTROCHI, D.A. Scale, multiscaling, remote sensing, and GIS. In: QUATTROCHI, D.A. \& GOODCHILD, M.F. (Editores). Scale in remote sense and GIS. Florida : CRC Lewis Publishers, 1997, p. 1-12.

LACOSTE, Y. De la géographie aux paysages. Paris: Armand Colin, 2003.

LAURELLI, E. Dinámicas mundiales e integración regional en espacios periféricos en los umbrales del siglo XXI. En: ZARATE, R. y ARTESI, L. (Editores). Dinámicas mundiales, integración regional y patrimonio en espacios periféricos. Rio Gallegos: UNPA, 2004, p. 7-21.

LAURELLI, E. y SCHWEITZER, A. La formación de regiones transfronterizas en el espacio de la Patagonia Austral. Revista de Estudios Regionales y Mercado de Trabajo, 2005, Vol. 1, p. 71-94.

MACHADO, L.O. Limites e fronteiras da alta diplomacia aos circuitos ilegais. Território, 2000, Vol. 5, № 8, p. 9-29.

MACHADO, L.O. Sistemas, fronteira e território. In: MACHADO, L.O. (Editora). Terra Limitanea: Atlas da Fronteira Continental do Brasil. Rio de Janeiro: Grupo RETIS/CNPq/UFRJ, 2002, p. 10-22.

MARSTON, S.A. The social construction of scale. Progress in Human Geography, v. 24, n. 2, p. 219$242,2000$.

MARTINS, J.S. Fronteira: a degradação do outro nos confins do humano. São Paulo: Editora Contexto, 2014.

MISIONES. Constitución de la Província de Misiones. Posadas, 1998.

MISIONES. Ley nº 3.631. Posadas, 1999.

OST, F. La nature hors la loi. Paris: La Découverte, 2003. 
PARAGUAY. Ley nº 3239/2007 de los recursos hídricos del Paraguay. Asunción, 2007.

PIRES DO RIO, G.A. Espaços protegidos transfronteiriços: patrimônio natural e territórios na bacia do Alto Paraguai. Revista Sustentabilidade em Debate, 2011, Vol. 2, № 1, p. 65-80.

REBORATTI, C.A. Fronteras agrarias en America Latina. Geocrítica, 1990, Vol. XV, No 87. Disponible en Internet: http://www.ub.edu/geocrit/geo87.htm

SMITH, N. Geografia, diferencia y políticas de escala. Terra Livre, 2002, Vol. 18, № 19, p. 127-146.

STEIMAN, R. Áreas Protegidas nas Zonas de Fronteira Internacional da Amazônia Brasileira. Rio de Janeiro: Universidade Federal do Rio de Janeiro,2008.

UNIÓN INTERNACIONAL PARA LA CONSERVACIÓN DE LA NATURALEZA (UICN). Bénéfices par-delà les frontières. In: Procès-verbaux du V Congrès mondial des parcs de I'UICN. 2005. Disponible en Internet:

http://www.nature-worldwide.info/downloads/UICN/benefits_beyond_bounderies-fr.pdf

UNIÓN INTERNACIONAL PARA LA CONSERVACIÓN DE LA NATURALEZA SUR (UICN). Áreas Protegidas en Latinoamérica: de Caracas a Durban. 2003. Disponible en Internet: http://data.UICN.org/ dbtw-wpd/edocs/2003-060.pdf

UNITED NATIONS ORGANIZATION FOR EDUCATION, SCIENCE AND CULTURE (UNESCO). Convention pour la protection du patrimoine mondial, culturel et naturel. 1972. Disponible en Internet: http://whc.unesco.org

WOLMER, W. Transboundary protected area governance: tensions and paradoxes. In: Transboundary Protected Areas in the Governance Stream of the $5^{\text {th }}$ World Parks Congress. Durban, 2003. 